\title{
EAI Endorsed Transactions

\section{Computational Analysis of Blood Flow Characteristics in an Aortic System with Abdominal and Left Common Iliac Aneurysm Pre- and Post-Stent Grafting}

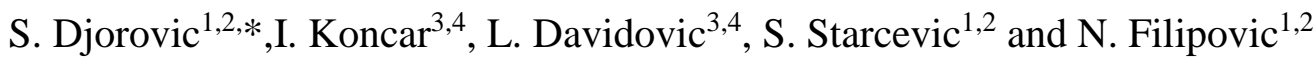 \\ ${ }^{1}$ Faculty of Engineering, University of Kragujevac, Kragujevac, Serbia \\ ${ }^{2}$ BioIRC Research and Development Center for Bioengineering, Kragujevac, Serbia \\ ${ }^{3}$ School of medicine, University of Belgrade, Belgrade, Serbia \\ ${ }^{4}$ Clinic for Vascular and Endovascular Surgery, Serbian Clinical Center, Belgrade, Serbia
}

\section{Abstract}

The aim of this study was to demonstrate how fluid dynamic parameters are affected by aortic geometry and flow condition in two cases. Case A included blood flow analysis in aortic system with abdominal aortic aneurysm and left common iliac aneurysm before stent graft placement, while in case B was included stent graft geometry, at the site of the aneurysms. An individual patient-specific geometry and a 3D finite element meshes were reconstructed, based on Computed tomography (CT) scan images. The analysis was performed using the possibilities of computational fluid dynamics. It uses numeric methods and algorithms for the simulation of blood flow by solving the Navier-Stokes equations on computational meshes. The computational simulations of cardiac cycles were performed for average blood properties and blood flow rate. The velocity field, pressure and shear stress, as main fluid dynamics parameters, were visualized and compared for cases A and B.

Keywords: computational fluid dynamics, blood flow, aortic aneurysm, stent graft.

Received on 11 August 2017, accepted on 09 October 2017, published on 28 February 2018

Copyright $@ 2018$ S. Djorovic et al., licensed to EAI. This is an open access article distributed under the terms of the Creative Commons Attribution licence (http://creativecommons.org/licenses/by/3.0/), which permits unlimited use, distribution and reproduction in any medium so long as the original work is properly cited.

doi: 10.4108/eai.28-2-2018.154145

\section{Introduction}

Abdominal aortic aneurysm (AAA) is a serious disease in both the US and Western Europe [1]. It requires an endovascular aneurysm repair (EVAR) as an alternative and minimally invasive treatment to standard open aneurysm repair [2]. Blood flow analyses in complete aorta, aortic aneurysms and stent grafts [3] belong to very intensive investigations of fluid mechanics that show the influence of blood flow conditions and geometry on the hemodynamic parameters. In this study we analyzed an individual male patient, aged 65, pre and post EVAR. The geometries of the complete aorta, and stent graft, were reconstructed from patient's CT scan images. Creation of

*Corresponding author. Email: smiljana@kg.ac.rs
3D models was based on finite element method (FEM). A 3D computational fluid dynamics simulations of the cardiac cycles for average blood properties and blood flow were performed using the software PAK-F [4]. This software has modules for steady and transient incompressible fluid flow. The modules are based on FEM and fundamental equations of viscous fluid flow.

The main aim of our study was to determine how the fluid dynamic parameters are affected by aortic geometry and flow condition in two cases. Case A included blood flow analysis in aortic system with abdominal aortic aneurysm and left common iliac aneurysm before stent graft placement, while in case B was included stent graft geometry, at the site of the aneurysms. The fluid dynamic parameters such as, velocity field, pressure and shear 
stress were compared in these two cases. The obtained results are presented and analyzed in this paper.

\section{Method}

\subsection{Geometrical models}

A set of DICOM images, with resolution of $512 \times 512$ $\mathrm{mm}$ and the slice increment of $0,625 \mathrm{~mm}$, were obtain using CT scanner and imported in the segmentation software which gives a highly accurate $3 \mathrm{D}$ model of the patient's anatomy. User can manipulate in three crosssections (coronal, sagittal and transversal). The stereolithography (STL) representation of the inner aortic wall set the boundary surface for the fluid domain. On the initial 3D models optimization of the surface meshes was performed.

The models were created for Cases $\mathrm{A}$ and $\mathrm{B}$. The difference between stent graft and aneurysmal geometry can be seen on Figure 1, while the other aortic regions were the same for both models (marked with A-G). An estimated size of proximal and distal (flared branch end) stent graft diameters were approximately $24 \mathrm{~mm}$ and 25 $\mathrm{mm}$, respectively, while graft branches had average diameters of $14 \mathrm{~mm}$. The volumetric models were generated using Femap software (Femap v.11, Siemens PLM Software, USA) in conjunction with our own software tools for the conversion of tetrahedral elements to eight-node brick elements. The datasets were imported into the software PAK-F for further computational simulations.

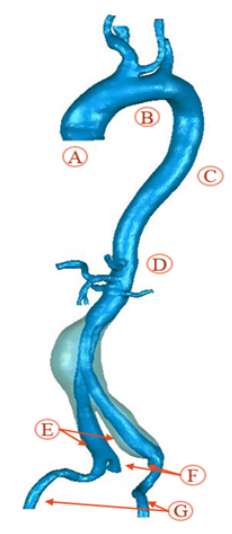

A. Ascending aorta

B. Aortic arch with roots of brachiocephalic trunk (BCT), left common carotid artery (LCA) and left subclavian artery (LSA)

C. Descending thoracic aorta

D. Descending abdominal aorta (with celiac, superior mesenteric and renal arteries)

E. Right and left common iliac arteries

F. Internal iliac arteries

G. External iliac arteries

Figure 1. Finite element mesh of the aortic system with markers (A-G)

\subsection{Numerical simulation}

In order to perform analysis which reflects the realistic human blood flow we used an average blood properties: a dynamic viscosity $(\mu)$ of $0,0035 \mathrm{~Pa} \cdot \mathrm{s}$ and a density $(\rho)$ of $1,050 \mathrm{~g} / \mathrm{cm}^{3}$ [5]. Flow is considered to be laminar, homogeneous (Newtonian) and viscous incompressible. The code used for this complex time-dependent and full 3D fluid flow was validated using the analytical solution for shear stress and velocities through a 3D curved tube [6], [7]. The 3D flow is governed by the Navier-Stokes equations and the continuity equation, which can be written as follows:

$$
\begin{gathered}
\rho\left(\frac{\partial v_{i}}{\partial t}+v_{j} \frac{\partial v_{i}}{\partial x_{j}}\right)=-\frac{\partial p}{\partial x_{i}}+\mu\left(\frac{\partial^{2} v_{i}}{\partial x_{j} \partial x_{j}}+\frac{\partial^{2} v_{j}}{\partial x_{j} \partial x_{i}}\right) \\
\frac{\partial v_{i}}{\partial x_{i}}=0
\end{gathered}
$$

where $v_{i}$ denotes the blood velocity in direction $x_{i}, p$ is the pressure, $\rho$ is the fluid density and $\mu$ is the dynamic viscosity. Summation is assumed on the repeated indices $i, j=1,2,3$. Equation (1) represents the balance of linear momentum, while (2) expresses the incompressibility condition. The total number of nodal points which formed the 3D eight-node elements was 379145 in Case A and 409395 in Case B, with three velocity components at all nodes. Penalty method was employed [6]. There were 40 steps with a constant time step of $0,025 s$ in each step of the simulation. Convergence criterion was reached for each time step, where the maximum number of available iterations was 15 .

For this study the boundary conditions consisted of: prescribed velocity at the root of the ascending aorta, zero-velocity at the rigid walls and physiological resistance pressure at the aortic outlets. At the aortic inlet, a parabolic flow velocity profile was used together with a pulsatile waveform (Figure 2) based on the measured data [8].

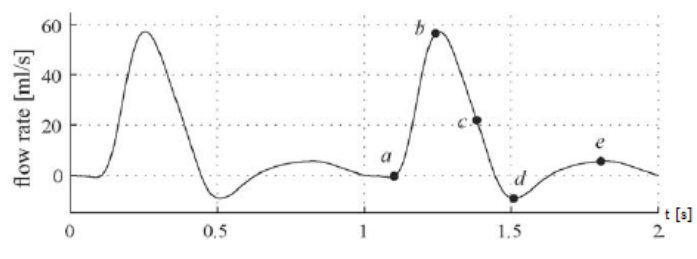

Figure 2. Prescribed blood flow rate [8], [9]: (a) enddiastole (b) peak-systole; (c) late-systole; (d) end-systole; (e) mid-diastole

\section{Results and discussion}

The main fluid dynamic parameters, such as velocity field, pressure and shear stress distribution are respectively presented for on Figure 3 (Case A) and Figure 4 (Case B). The models are presented from anterior view at peak systole moment, as the worst-case scenario, which is reached after about $0,3 s$ of the simulations. 
In case of velocity field distribution (Units: $\mathrm{m} / \mathrm{s}$ ) for both models, higher velocities dominate in the ascending aorta, aortic arch with roots of BCT, LCA and LSA, as well as in the smaller branches of the abdominal aorta. Decreased velocity can be seen in the abdominal aneurysm and left common iliac aneurism (Case A), while in the stent graft (Case B) velocity has the highest value at the graft bifurcation, and gradually decreases going towards lower graft parts, up to the ending of external iliac arteries. At the internal iliac arteries can be seen slightly higher velocity. Velocity distribution directly affects pressure and shear stress distribution.

Pressure (Units: $\mathrm{Pa}, 1 \mathrm{~Pa}=0,0075 \mathrm{~mm} \mathrm{Hg}$ ) is equal at the ascending aorta and aortic arch with roots of BCT, LCA and LSA for both cases, and it reaches the maximal blood pressure which corresponds to the maximal human blood pressure of around $125 \mathrm{~mm} \mathrm{Hg}$. The pressure decreases distal to the ascending aorta, with slightly variations in Cases A and B. Decreased pressure can be seen in the abdominal aneurysm and left common iliac aneurism (Case A), while in the stent graft (Case B) pressure has the highest value at the graft beginning, and gradually decreases going towards lower graft parts, up to the ending of external and internal iliac arteries. Blood pressure has a dominant influence on drag forces [10], so that an increase in mean blood pressure results in linearly proportional increase in drag forces magnitude. Also, drag forces depend on the anatomically curved configuration of the stent graft, anterior/posterior angle and inlet crosssectional area. Drag forces analysis may help determine stent graft migration [11] that will be focus of our future studies.

The highest shear stress (Units: $\mathrm{Pa}$ ) is located at aortic arch with root of BCT, LCA and LSA in both cases. A jet of fluid, created during systole provides high velocity and high shear stress at the stent graft bifurcation (Case B). Low shear stress is observed along the zones in the aneurysmal parts (Case A), where blood velocity has low values. It makes condition for bigger aneurysmal sac creation and its further growth, so that is reasonable why the stent graft had to be implanted.

This analysis was built on experiences of other groups having performed CFD simulations of blood flow in the human aorta [3], [12]. CFD simulations were performed, with the aim to investigate how the flow field is affected by aortic and stent graft geometry and flow condition. From the technical point of view, this study was performed to validate the algorithm with eight-node finite element and penalty formulation for complex pulsatile fluid flow in the human aorta.

Finally, this study has a several limitations. There were several assumptions in the CFD simulation in order to simplify the simulations. We assumed that there were not blood flow in the aneurysmal sac after stent graft implantation. We did not have the measured blood flow for this specific patient, so we took the average values. Also, we did not consider wall properties and fluidstructure interaction [5], [9], [11] which have important role in mechanisms of aneurysmal growth and rupture, as well as in the stent graft migration and durability.
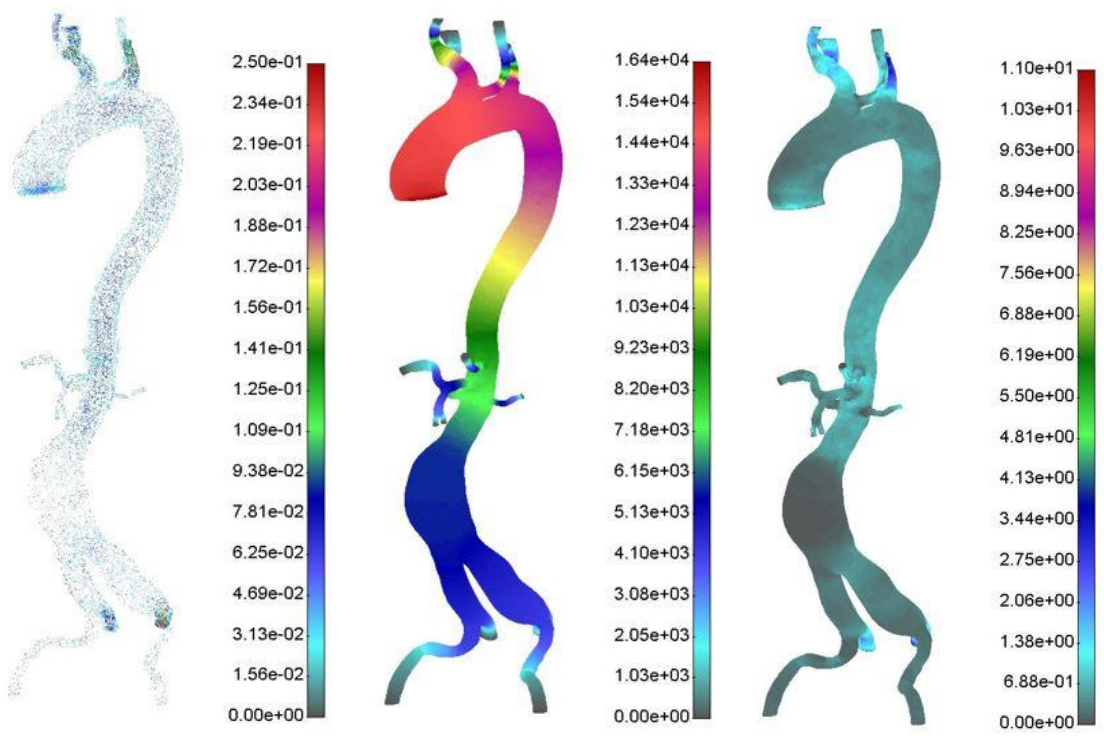

Figure 3. Case A: Velocity field (Units: $\mathrm{m} / \mathrm{s}$ ), Pressure (Units: $\mathrm{Pa}$ ) and Shear stress (Units: $\mathrm{Pa}$ ) distribution, respectively 

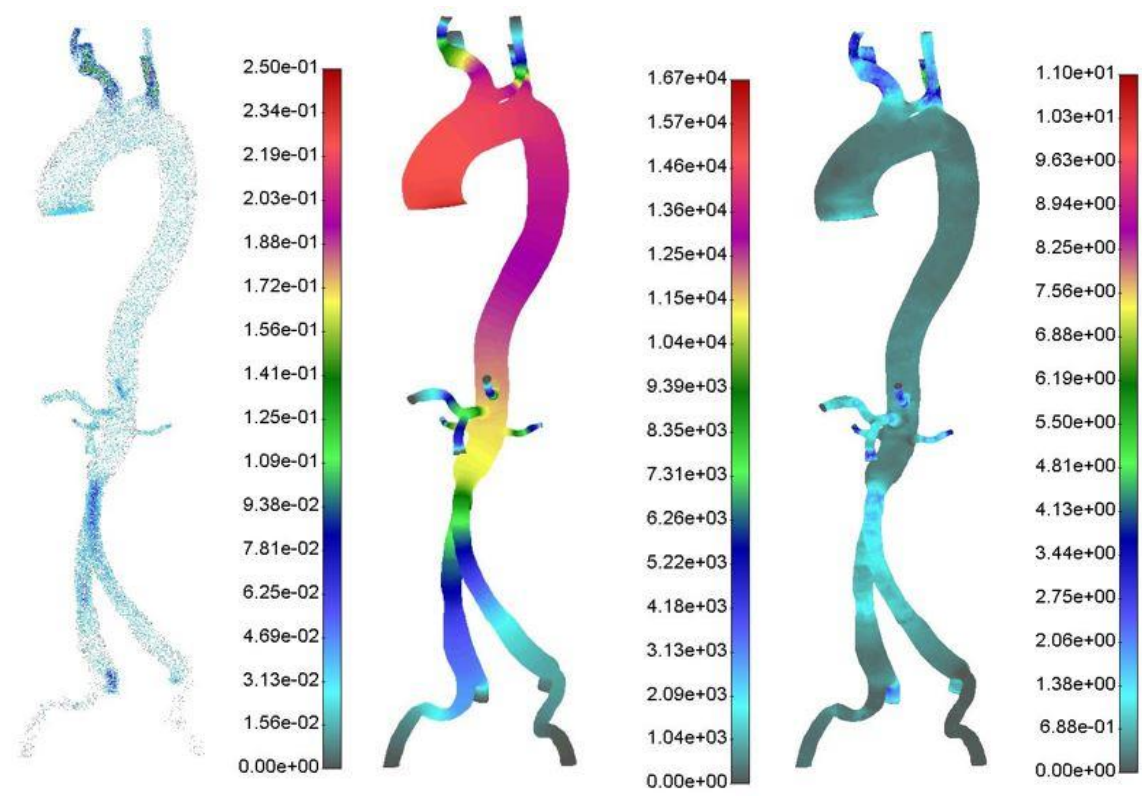

Figure 4. Case B: Velocity field (Units: $\mathrm{m} / \mathrm{s}$ ), Pressure (Units: $\mathrm{Pa}$ ) and Shear stress (Units: $\mathrm{Pa}$ ) distribution, respectively

\section{Conclusion}

After presented results, the stent graft implantation was reasonable and in order to prevent further aneurysmal growth and rupture. Certain geometrical factors have an influence on blood circulation and distribution of different hemodynamic parameters. Beside all benefits which aortic endovascular approach takes with it, various problems are still present, such as stent graft migration, different types of endoleaks and stent mechanism breakage. These phenomena depend on appropriate selection of stent graft type and dimensions which correlate with stent graft durability. In that order, this will be an objective of our future studies. Our knowledge about local anatomical and fluid dynamic conditions for abdominal aortic stent implantation and placement can be additionally improved by CFD as a highly promising technique.

\section{References}

[1] Brown, P.M., Zelt, D.T., Sobolev, B. (2003) The Risk of Rupture in Untreated Aneurysms: The Impact of Size, Gender, and Expansion Rate. J. Vasc. Surg. 37(2): 280284.

[2] Prinssen, M., Verhoeven, E., Buth, J., et al. (2004) A Randomized Trial Comparing Conventional and Endovascular Repair of Abdominal Aortic Aneurysms. $N$. Engl. J. Med. 351(16): 1607-1618.

[3] Frauenfelder, T., Lotfey, M., Boehm, T., Wildermuth, S. (2006) Computational Fluid Dynamics: Hemodynamic Changes in Abdominal Aortic Aneurysm After Stent-graft Implantation. Cardiovasc. Intervent. Radiol. 29(4): 613623.

[4] Kojic, M., Filipovic, N., Zivkovic, M, Slavkovic, R., Grujovic, N. (1999) PAK-F, Program for FE Analysis of Fluid Flow with Heat Transfer, Users Manual. Faculty of Mechanical Engineering of Kragujevac-Laboratory for Engineering Software, Kragujevac, Serbia.
[5] Gao, F., Watanabe, M., Matsuzawa, T. (2006) Stress Analysis in a Layered Aortic Arch Model Under Pulsatile Blood Flow. BioMed. Eng. Online. 5:25.

[6] Filipovic, N., Misailovic, S., Tsuda, A., Kojic, M. (2006) An Implicit Algorithm within the Arbitrary LagrangianEulerian Formulation for Solving Incompressible Fluid Flow with Large Boundary Motions. Comp. Meth. Appl. Mech. Eng. 195(44-47): 6347-6361.

[7] Kojic, M., Filipovic, N., Stojanovic, B., Kojic, N. (2008) Computer Modeling in Bioengineering: Theoretical Background, Examples and Software, (Wiley, England).

[8] Olufsen, M.S., Peskin, C.S., KIM, W.Y., Pedersen, E.M., NADiM, A., LARSEN, J. (2000) Numerical Simulation and Experimental Validation of Blood Flow in Arteries with Structured-Tree Outflow Conditions. Ann. Biomed. Eng. 28(11): 1281-1299.

[9] Wolters, B.J., Rutten, M.C., Schurink, G.W., Kose, U., de Hart, J., VAN DE Vosse, F.N. (2005) A Patient-Specific Computational Model of Fluid-Structure Interaction in Abdominal Aortic Aneurysms. Med. Eng. Phys. 27(10): 871-883.

[10] Morris, L., Delassus, P., Walsh, M., McGloughlin, T. (2004) A Mathematical Model to Predict the In Vivo Pulsatile Drag Forces Acting on Bifurcated Stent Grafts Used in Endovascular Treatment of Abdominal Aortic Aneurysms (AAA). J. Biomech. 37(7): 1087-1095.

[11] Li, Z., Kleinstreuer, C., Farber, M. (2005) Computational Analysis of Biomechanical Contributors to Possible Endovascular Graft Failure. Biomech. Model. Mechanobiol. 4(4): 221-234.

[12] Sun, Z., Chaichana, T., SANGworasil, M, Tungjitkusolmun. S. (2008) Computational Fluid Analysis of Blood Flow Characteristics in Abdominal Aortic Aneurysms Treated with Suprarenal Endovascular Grafts. In: 13th International Conference on Biomedical Engineering, Singapore, 3-6 December (Berlin, Heidelberg: Springer), IFMBE Proceedings, 23: 17281732. 